\title{
Using Near-Field Light Sources to Separate Illumination from BRDF.
}

Jeroen Put

jeroen.put@uhasselt.be

Nick Michiels

nick.michiels@uhasselt.be

Philippe Bekaert

philippe.bekaert@uhasselt.be
Hasselt University - tUL - iMinds

Expertise Centre for Digital Media

Wetenschapspark 2

3590 Diepenbeek, Belgium

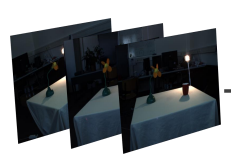

input

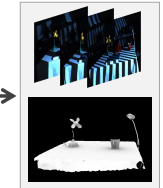

reconstruction

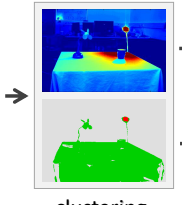

clustering

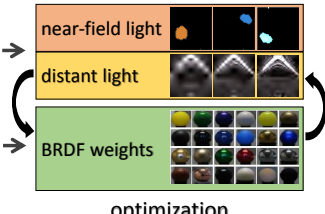

optimization

Figure 1: Schematic overview of our method.

Simultaneous estimation of lighting and BRDF (Bidirectional Reflectance Distribution Function) from multi-view images is an interesting problem in computer vision. It allows for exciting applications, such as flexible relighting in post-production, without recapturing the scene. The ability to alter scenes after they have been filmed has the potential to greatly reduce the number of costly recapturing iterations. Unfortunately, the estimation problem is made difficult because lighting and BRDF have closely entangled effects in the input images. This paper presents an algorithm to support both the estimation of distant and near-field illumination. Previous techniques are limited to distant lighting. We contribute by proposing an additional factorization of the lighting, while keeping the rendering efficient and additional data compactly stored in the wavelet domain. We reduce complexity by clustering the scene geometry into a few groups of important emitters and calculate the emitting powers by alternately solving for illumination and reflectance. We demonstrate our work on a synthetic and real datasets and show that a clean separation of distant and near-field illumination leads to a more accurate estimation and separation of lighting and BRDF.

The input of our algorithm is a sparse set of (usually 5-16) different views of a scene with unknown reflectance, captured under unknown illumination. This makes our method very broadly applicable. The outputs of our algorithm are the estimated wavelet coefficients of the distant lighting environment map, the estimated emitting powers for every emitting cluster in the scene and a set of per-vertex material weights that determine a mixture of BRDF models [3].

We can summarize our contributions in a few key points:

1. A factorization of illumination in distant and near-field lighting. This allows us to model local lighting effects, many of which are ignored by current methods (Figure 2).

2. A clean separation of distant and near-field lighting allows more accurate estimation of illumination and materials in a scene and permits the manipulation of local light sources. It also allows using knowledge of the light sources in a scene. For example, light source positions can be identified by a HDR clustering procedure and help improve the estimation of other factors.

3. We formulate the problem so that the wavelet coefficients of the distant lighting environment map and the emitting powers of the near-field lighting can be solved together. Any standard quadratic programming framework can be used to solve this problem robustly and efficiently.

4. We propose the use of clustering to group the most significant emitting triangles into dominant light sources. This grouping constrains the solver to more plausible solutions. We make the assumption that the distribution of light is roughly uniform along the emitting surface.

We propose factorizing illumination in distant and near-field lighting components. The distant illumination is expressed in wavelet coefficients [2], which are estimated directly. Then, we further factorize the

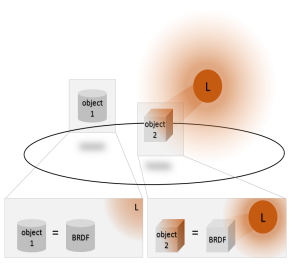

(a)

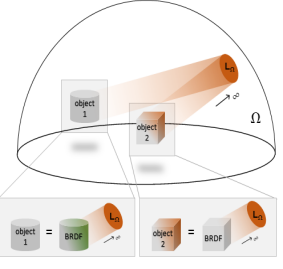

(b)

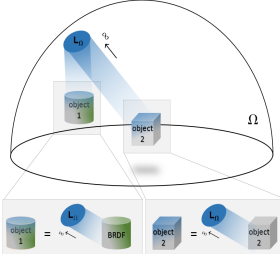

(c)
Figure 2: (a) A scene with 2 objects and a red local light source $L$. (b) Current techniques model local lighting effects with distant light sources at infinity. This causes erroneous correction colors (shown here as green) in the BRDF. (c) Relighting reveals the wrongly estimated materials.

near-field lighting term at every vertex. Rendering with the factorized representation is equivalent to adding an extra double product integral of the BRDF and the irradiance maps to the rendering equation. Because Haar wavelets are an orthogonal basis, this double product integral term reduces to a simple dot product calculation [2]:

$$
B\left(v, \omega_{0}\right)=\underbrace{\sum_{k} \sum_{l} \sum_{m} C_{k l m} \rho_{k} V_{l} \widetilde{L}_{m}}_{\text {distant lighting }}+\underbrace{\sum_{c} \sum_{k} \rho_{k} \cdot\left(I_{c} P_{c}\right)}_{\text {near-field lighting }}
$$

For every cluster $c$, visible over the hemisphere of vertex $v$, its irradiance map $I_{c}$ is multiplied by the estimated power $P_{c}$. Finally, it is multiplied by the BRDF $\rho$ at $v$. At the end, all this information is assembled in bilinear equations and finally into matrix form. The unknowns are then solved for with a quadratic programming solver. Figure 1 gives a summary of our algorithmic pipeline.

Figure 3 shows the improvement that our new factorization offers. By taking near-field lighting effects into account, material and lighting properties of objects can be more faithfully recovered. (a)

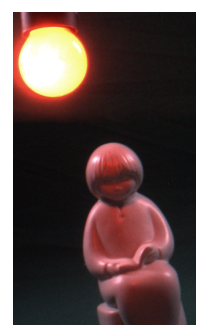

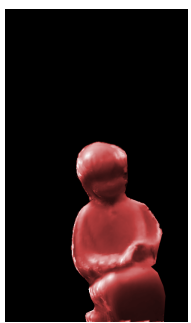

(b)

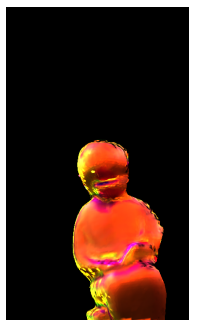

(c)

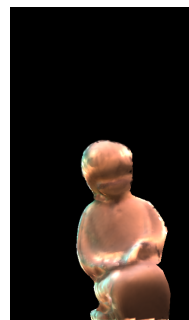

(d)
Figure 3: A white statuette under a red light source dataset. (a) One of the input images. (b) Reconstruction after optimization. (c) The technique of Haber et al. [1] reveals compensation colors in materials when rendered under white lighting. (d) Material estimated by our technique rendered under white lighting.

[1] T. Haber, C. Fuchs, P. Bekaert, H.-P. Seidel, M. Goesele, and H.P.A. Lensch. Relighting objects from image collections. In CVPR 2009, pages 627-634, June 2009.

[2] Ren Ng, Ravi Ramamoorthi, and Pat Hanrahan. Triple product wavelet integrals for all-frequency relighting. ACM Trans. Graph., 23(3):477-487, August 2004. ISSN 0730-0301.

[3] R. Peter Weistroffer, Kristen R. Walcott, Greg Humphreys, and Jason Lawrence. Efficient basis decomposition for scattered reflectance data. In Proceedings of the 18th Eurographics conference on Rendering Techniques, EGSR'07, pages 207-218, 2007. ISBN 978-3905673-52-4. 